\title{
Improving the Performance of ITO Thin Films by Coating PEDOT:PSS
}

Tsai-Cheng Li' and Rwei-Ching Chang ${ }^{1, *}$

1 Department of Mechanical and Computer-Aided Engineering, St. John's University, New Taipei City, 25135, Taiwan \# Corresponding Author / E-mail: rcc@mail.sju.edu.tw, TEL: +886-2-28013131, FAX: +886-2-28013143

KEYWORDS: ITO thin films, PEDOT:PSS, Electric resistance, Optical transmittance, Adhesion

\begin{abstract}
This work studies the effect of PEDOT:PSS on electrical, optical and mechanical properties of ITO glass. Various thickness PEDOT:PSS films are deposited on ITO glass by spin coating. The sheet resistance, transmittance, surface roughness, residual stress, friction, and critical load of the coated specimens are tested in sequence. We find that PEDOT:PSS films not only improve the electric performance but also increase the adhesion of ITO glass. The experimental results show that the PEDOT:PSS film improves the surface roughness and then results in a lower electric resistance. It also increases the adhesion and reduces the residual stress of the ITO films. Furthermore, only 1\% transmittance is lost due to the PEDOT:PSS film, showing the potential in the applications of organic photovoltaic cells and light-emitting diodes.
\end{abstract}

Manuscript received: June 22, 2014 / Revised: September 1, 2014 / Accepted: September 15, 2014

\section{Introduction}

With high optical transmittance and low electric resistance, indium tin oxide (ITO) thin films on glass substrates have been broadly applied as conductive electrodes for various displays and photovoltaic devices. ${ }^{1,2}$ While ITO is the most popular transparent conducting film (TCF) in inorganic materials, PEDOT:PSS is the most promising candidate in organic TCFs. ${ }^{3-5}$ In the applications of organic photovoltaics (OPVs) and organic light-emitting diodes (OLEDs), PEDOT:PSS is often coated on the ITO, where the ITO is utilized as an anode and the PEDOT:PSS is as hole conducting layer., ${ }^{6,7}$ OPVs or OLEDs with a PEDOT:PSS layer on top of an ITO anode have longer lifetime and higher photovoltaic performance than those using ITO anode alone. ${ }^{8}$ The improvement in device stability can be attributed to the ability of the polymer coating to clean the anode surface that substantially slows down device degradation. The photovoltaic performance improved because the conducting polymer may produce a smaller energy barrier that lowers the turn-on voltage of the electronic device. ${ }^{9}$

The structure, morphology and electro-optical characteristics of the transparent anode hole conducting layers play an important role on the final device performance. ${ }^{10-12}$ Except electrical and optical properties, mechanical properties also play an important role in thin film devices. Large deformation and debonding of thin films will affect the performance and stability of electronic devices. The elastic modulus and hardness of the transparent anode and hole conducting layers provide basic mechanical support in the layer stack. ${ }^{13}$ However, residual stresses appearing in the deposited thin films are unavoidable. The mismatch of lattice and thermal expansion between the thin film and substrate induces certain amount of stresses. ${ }^{14}$ Heat accumulates during the usage of OPVs and OLEDs resulting in thermal stresses. Stresses in thin films can be either beneficial or detrimental to the performance, which may improve the film stiffness or distort its structures, resulting in cracking in the case of tensile stresses or buckling in compressive stresses. Moreover, delamination reduces the lifetime and reliability of deposited films. The adhesion between inorganic and organic films is a crucial factor that needs to improve in component fabrication. ${ }^{15}$ However, study of the mechanical properties of organic electronic devices is still rare in literature.

To improve the performance of the organic electronic devices, it is important to get a better understanding of the interaction between PEDOT:PSS and ITO films. Therefore, this work focuses on characterizing the properties of PEDOT:PSS coated on ITO glass. The influence of various thickness PEDOT:PSS on the structure, morphology and electro-optical characteristics of ITO glasses has been analyzed. Furthermore, the mechanical properties of the thin film/ substrate structures, including elastic modulus, hardness, residual stress, and critical load, are first reported. All test results are compared with those of the bare ITO glass. 


\section{Experimental Details}

Various thickness PEDOT:PSS (Clevios AI4083) films are deposited on a $20 * 30 \mathrm{~mm}$ ITO glass by spin coating. All the ITO glass substrates are cleaned in an ultrasonic bath and treated by $\mathrm{O}_{2}$ plasma. Three thicknesses of the PEDOT:PSS films are considered, including 30,40 , and $50 \mathrm{~nm}$. The thickness of each layer are controlled by the spin speed and confirmed by a Kosaka ET4000 surface profiler.

A grazing-incidence X-ray diffraction (XRD) machine (PANalytical X'Pert PRO MPD) with a copper anode $(\lambda=0.154 \mathrm{~nm})$ is utilized in the microstructure analysis. Residual stresses of thin films are measured by using the $\sin ^{2} \psi$ method of XRD in this work. The basic concept of the XRD analysis is that the lattice spacing varies with the orientation of the lattice planes with respect to the loading direction. ${ }^{16,17}$ In XRD analysis, Bragg's law gives ${ }^{16}$

$$
n \lambda=2 d \sin \theta
$$

where $n=1,2,3, \ldots, \lambda$ is the wavelength, $d$ denotes the lattice spacing, and $\theta$ is the diffraction angle. According to the variation of the lattice spacing, the elastic strain can be defined as

$$
\varepsilon=\left(d-d_{0}\right) / d_{0}
$$

where $d_{0}$ is the strain-free lattice spacing. Note that the lattice spacing is measured in the direction of the scattering vector, which decreases as the lattice spacing planes parallel to a tensile stress and increases as its planes are normal to a the tensile loading direction. For elastically isotropic crystallites, Hooke's law relating the mechanical strain tensor $\varepsilon_{i j}$ to the mechanical stress tensor $\sigma_{k l}$ is given as ${ }^{17}$

$$
\varepsilon_{i j}=S_{i j k l} \sigma_{k l}=\left[S_{1} \delta_{i j} \delta_{k l}+\frac{1}{4} S_{2}\left(\delta_{i k} \delta_{j l}+\delta_{i l} \delta_{j k}\right)\right] \sigma_{k l}
$$

where $S_{i j k l}$ is the compliance tensor. Summation convention over the dummy indices is adopted throughout the paper. The only two independent components $S_{1}$ and $S_{2}$ are defined as

$$
S_{1}=-v / E, S_{2}=2(1+v) / 2
$$

which relate to the elastic modulus $E$ and Poisson's ratio $v$. Consequently, the strain tensor in the diffraction direction can be expressed as

$$
\begin{gathered}
\varepsilon_{\varphi \psi}=\frac{1}{2} S_{2} \sin ^{2} \psi\left[\sigma_{11} \cos ^{2} \varphi+\sigma_{12} \sin 2 \varphi+\sigma_{22} \sin ^{2} \varphi\right] \\
+\frac{1}{2} S_{2}\left[\sigma_{13} \cos \varphi \sin 2 \psi+\sigma_{23} \sin \varphi \sin 2 \psi+\sigma_{33} \cos ^{2} \psi\right] \\
+S_{1}\left(\sigma_{11}+\sigma_{22}+\sigma_{33}\right)
\end{gathered}
$$

For a rotationally symmetric biaxial stress state, it gives $\sigma_{11}=\sigma_{22}=\sigma, \sigma_{12}=\sigma_{13}=\sigma_{23}=\sigma_{33}=0, \varphi=0$

Then, the strain can be reduced to

$$
\varepsilon_{\psi}=\left(2 S_{1}+\frac{1}{2} S_{2} \sin ^{2} \psi\right) \sigma
$$

Therefore, the biaxial stress or the residual stress $\sigma$ can be obtained from the slope of the straight line in the plot of $\varepsilon_{\psi}$ versus $\sin ^{2} \psi 17$. This method is also called the $\sin ^{2} \psi$ method and will be used in the following discussion.

Typical nanoindentation experiments are performed by using a Hysitron Triboindenter fitted with a standard Berkovich indenter. The loading time, holding time, and unloading time of the indentation tests are all $5 \mathrm{sec}$. The peak load is controlled at $250 \mu \mathrm{N}$. A standard analysis to determine the hardness and elastic modulus from the unloading loaddepth curve is conducted according to the Oliver and Pharr method. ${ }^{18}$ The hardness $H$ can be defined as

$$
H=P_{\max } / A
$$

where $P_{\max }$ represents the peak load and $A$ is the projected contact area. The hardness is the mean pressure that a material can support under load. The relation between the load $(P)$ and the depth $(h)$ can be represented as ${ }^{18}$

$$
P=\alpha\left(h-h_{f}\right)^{m}
$$

where $a$ is an indent constant, $m$ depends on the geometry of the indenter, and $h_{f}$ indicates the final depth. The elastic modulus of the indented sample can be inferred from the initial unloading contact stiffness, $S=d P / d h$, i.e., the slope of the initial portion of the unloading curve. Based on the indentation analysis developed by Snedden, ${ }^{19}$ the relation between the contact stiffness, contact area, and elastic modulus can be derived as

$$
S=2 \beta \sqrt{\frac{A}{\pi}} E_{r}
$$

where $\beta$ is a constant that depends on the geometry of the indenter, $\beta=1.034$ for Berkovich indenter, and $E_{r}$ is the reduced elastic modulus, which accounts for the elastic deformation occurs in both the sample and the indenter. Hence, $E_{r}$ is given by

$$
\frac{1}{E_{r}}=\frac{1-v^{2}}{E}+\frac{1-v_{i}^{2}}{E_{i}}
$$

where $E_{i}$ and $v_{i}$ are the elastic modulus and Poisson's ratio of the indenter. The elastic modulus $E$ of the sample can be determined by Eq. (10) with assuming a proper Poisson's ratio $v$. The standard analysis of nanoindentation is called Oliver and Pharr method. ${ }^{18}$ Then, following the Oliver and Pharr method, the hardness and elastic modulus can be determined by Eqs. (7) and (10).

Electrical sheet resistance of the thin films was examined by using a four-point probe (QUATEK 5610Y/QT-50). Optical transmittance of the specimens was measured by using an UV/VIS/NIR spectrophotometer (BWTEK BTC112) with normal incidence in the visible wavelength ranged from 400 to $800 \mathrm{~nm}$, taking the air as reference. In addition, the surface roughness was tested by a white light interferometer (BMT WLI LAB).

The critical load of micro scratch testing can be used to represent the adhesion of thin films. In this work, the micro scratch is conducted by a Rhesca CSR- $02 \mathrm{~F}$ thin film scratch tester. A $5 \mu \mathrm{m}$ diameter stylus is utilized, and the stage angle is tilt $1^{\circ}$ with speed $10 \mu \mathrm{m} / \mathrm{s}$. In the 
micro scratch, the specimen is mounted on the tilt stage which moves horizontally to push up the stylus on the top end of the sensor. A load, as a consequence, is applied to the surface of the specimen by the stiffness and strain of the cantilever stylus. The cartridge of the micro scratch tester oscillates parallel to specimen surface at fixed amplitude acted by the build-in actuator. The friction between the specimen and the stylus causes relative movement and then generates electric output signals. As long as the specimen surface is not damaged, the sensor can generate output signals proportional to the stylus velocity. As the load reaches the critical value, some scratch noises are generated due to the destruction or rough surface of the sample film, which are reflected and observed as output signals. ${ }^{20}$

\section{Results and Discussion}

In order to confirm the crystal phase of ITO, XRD analysis is performed. Fig. 1 shows the XRD patterns of ITO glass with various thickness PEDOT:PSS films. Five XRD peaks, (211), (222), (400), (441), and (622), of ITO are positively identified, ${ }^{14}$ showing the preferred orientation (222) texture at $2 \theta=30.0^{\circ}$ for all cases. ${ }^{21}$ The detail values of $2 \theta$, intensity, and Full width at half maximum (FWHM) of the preferred orientation (222) are listed in Tables . No significant changes of the micro structures have been found after adding the PEDOT:PSS films, meaning no chemical reaction occurs between PEDOT and ITO.

The optical transmittance of ITO glass with various thickness PEDOT:PSS films is shown in Fig. 2. It shows the average transmittance of the ITO glass without PEDOT:PSS film is $91.86 \%$, and then decreases to $91.20,91.09$, and $90.49 \%$ as adding 30,40 , and

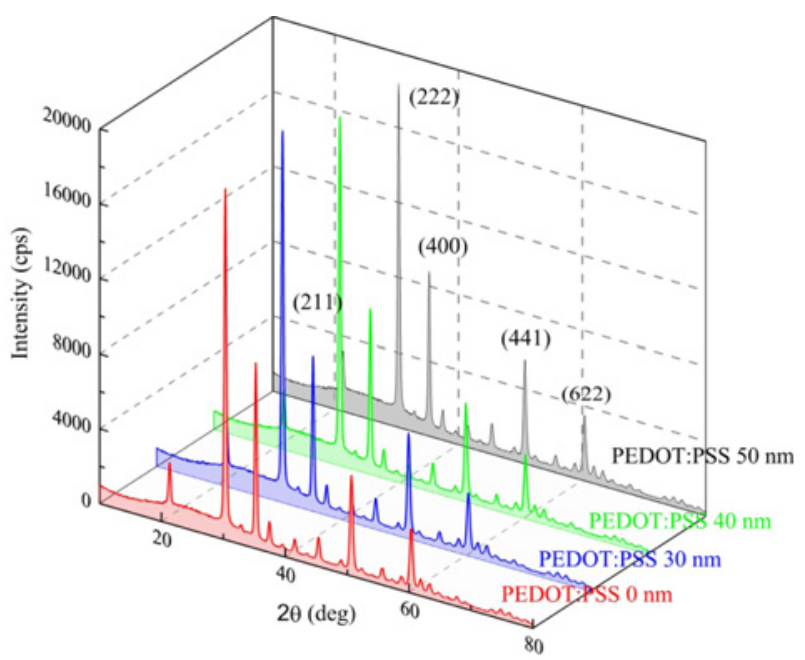

Fig. 1 XRD patterns of ITO glass with various thickness PEDOT:PSS films

Table 1 XRD results of peak (222) of ITO/Glass with various thickness PEDOT:PSS films

\begin{tabular}{ccccc}
\hline & $\begin{array}{c}\text { Without } \\
\text { PEDOT:PSS }\end{array}$ & $30 \mathrm{~nm}$ & $40 \mathrm{~nm}$ & $50 \mathrm{~nm}$ \\
\hline $2 \theta(\mathrm{deg})$ & 30.31 & 30.32 & 30.32 & 30.32 \\
\hline Intensity (cps) & 18755 & 19867 & 18648 & 18376 \\
\hline FWHM (deg) & 0.43 & 0.43 & 0.43 & 0.43 \\
\hline
\end{tabular}

$50 \mathrm{~nm}$ thick PEDOT:PSS films, respectively. The visible transmittance of the ITO glass is $95 \%$ at $500 \mathrm{~nm} .^{21}$ It shifts to $550 \mathrm{~nm}$ after adding the PEDOT:PSS films. The transmittance of ITO glass with 30 and $40 \mathrm{~nm}$ thick PEDOT:PSS films are even higher than the bare ITO glass. The transmittance shift may be the thickness effect because the thickness of ITO is $120 \mathrm{~nm}$ and the PEDOT:PSS arrives to $30-50 \mathrm{~nm}$. However, The PEDOT:PSS film slightly decreases $1 \%$ transmittance of the ITO glasses, illustrating PEDOT:PSS has good transparent performance.

Fig. 3 shows the residual stress of ITO glasses with various thickness PEDOT:PSS films. Based on the biaxial stress model shown in Eq. (6), the residual stress of the ITO thin film can be determined from the XRD results. Fig. 3 shows all the residual stresses for all cases are compressive. The residual stress of the ITO glass without PEDOT:PSS film is $2147.7 \mathrm{MPa}$, and then decreases to $1792.6,1770.8$, and $1757.3 \mathrm{MPa}$ as adding 30,40 , and $50 \mathrm{~nm}$ thick PEDOT:PSS films, respectively. It illustrates the PEDOT:PSS film deposited by spin coating provides a tensile stress which reduces the compressive

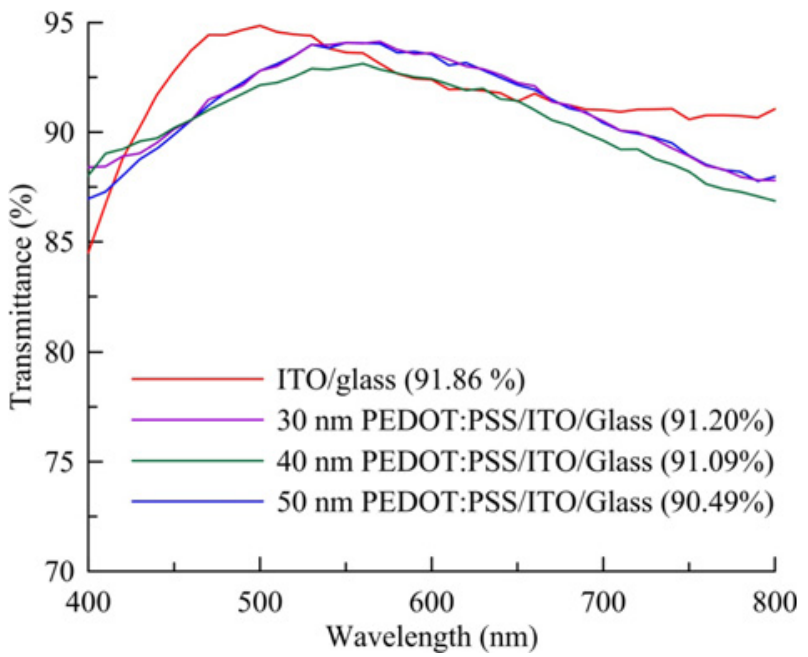

Fig. 2 Transmittance of ITO glass with various thickness PEDOT:PSS films

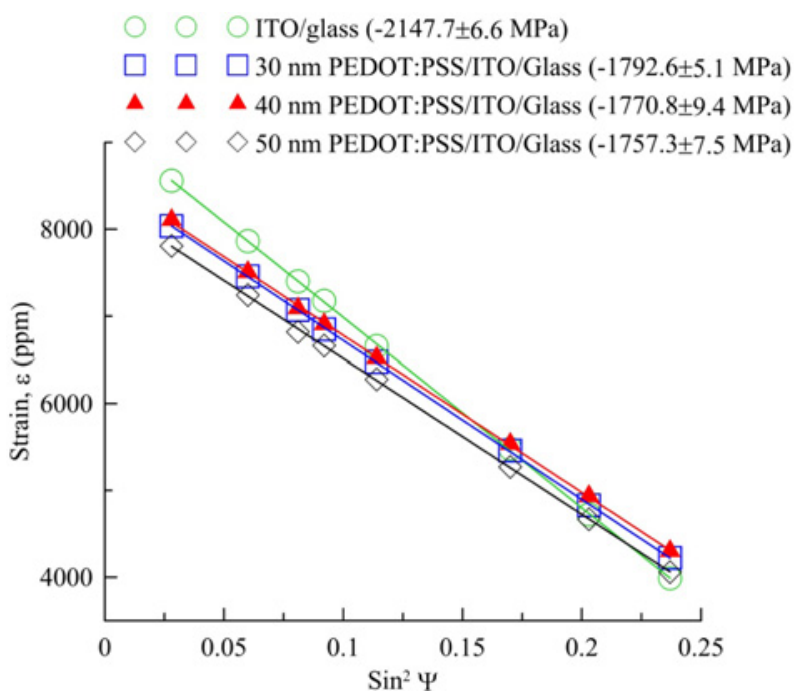

Fig. 3 Residual stresses of ITO glass with various thickness PEDOT:PSS films 
residual stress of the ITO film. Consequently, the residual stress decreases as the PEDOT:PSS thickness increases. In average, the PEDOT:PSS film decreases $17 \%$ residual stress of the ITO glass.

The load-depth curves of ITO glasses with various PEDOT:PSS thicknesses measured by nanoindentation are shown in Fig. 4, where the maximum load $250 \mu \mathrm{N}$ is applied. It illustrates the load-depth curves shift to right as the PEDOT:PSS films add, meaning the indentation probe is easier to press into the specimen's surface. The residual indentation depth of the ITO glass without PEDOT:PSS film is $5 \mathrm{~nm}$, and then increases to $40,55,65 \mathrm{~nm}$ as adding 30,40 , and $50 \mathrm{~nm}$ thick PEDOT:PSS films, respectively. The elastic reduced modulus and hardness of ITO glasses with various thickness PEDOT:PSS films measured by nanoindentation are listed in Table 2. The results show that the reduced modulus of the ITO glass without PEDOT:PSS film is $153.2 \mathrm{GPa}$, and then largely decreases to 77.23 , 76.60 , and $79.33 \mathrm{GPa}$ as adding 30,40 , and $50 \mathrm{~nm}$ thick PEDOT:PSS films, respectively. The hardness is $20.30 \mathrm{GPa}$, and decreases to 2.12 , 2.88 , and $1.58 \mathrm{GPa}$, similarly. It indicates that the PEDOT:PSS coating reduces the elastic modulus and hardness of the ITO film, because the mechanical properties of the polymeric coating are much smaller than the oxide conductive films. No discontinuities are found in the loading or unloading segment, showing neither twinning nor pressure-induced phase transformation is involved in the indentation process.

Fig. 5 and 6 show the micro scratch results of ITO glasses with

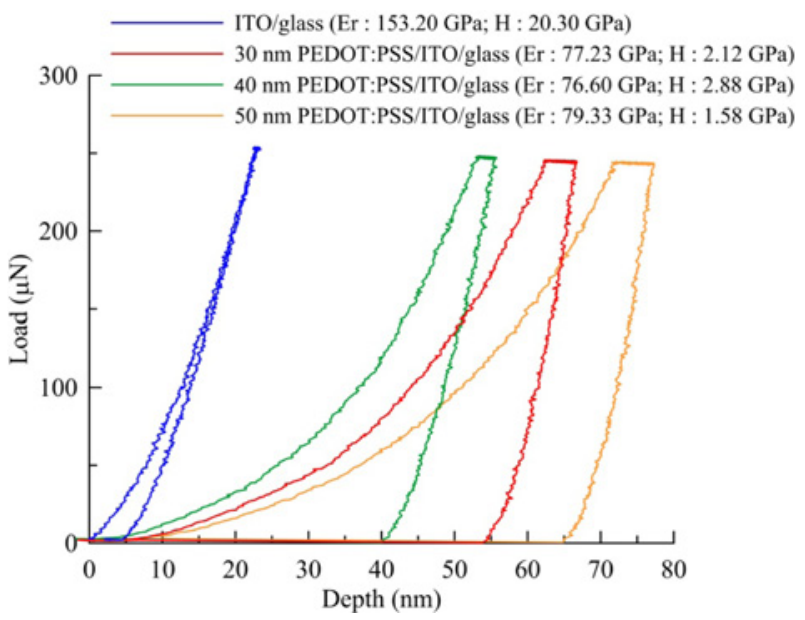

Fig. 4 Load-depth curve of ITO glass with various thickness PEDOT:PSS films

Table 2 Experimental results of ITO/Glass with various thickness PEDOT:PSS films

\begin{tabular}{ccccc}
\hline & $\begin{array}{c}\text { Without } \\
\text { PEDOT:PSS }\end{array}$ & $30 \mathrm{~nm}$ & $40 \mathrm{~nm}$ & $50 \mathrm{~nm}$ \\
\hline Transmittance (\%) & 91.86 & 91.2 & 91.09 & 90.49 \\
\hline $\begin{array}{c}\text { Residual stress } \\
(\mathrm{MPa})\end{array}$ & -2147.7 & -1792.6 & -1770.8 & -1757.3 \\
\hline $\begin{array}{c}\text { Reduced modulus, } \\
\mathrm{Er}(\mathrm{GPa})\end{array}$ & 153.2 & 77.23 & 76.60 & 79.33 \\
\hline Hardness, H $(\mathrm{GPa})$ & 20.30 & 2.12 & 2.88 & 1.58 \\
\hline Critical load $(\mathrm{mN})$ & 166.0 & 172.3 & 182.3 & 192.3 \\
\hline $\begin{array}{c}\text { Surface roughness, } \\
\mathrm{Ra}(\mathrm{nm})\end{array}$ & 11.0 & 8.62 & 6.26 & 5.36 \\
\hline Sheet resistance $(\Omega / \square)$ & 13.77 & 5.28 & 4.78 & 4.35 \\
\hline
\end{tabular}

various thickness PEDOT:PSS films. The scratch distance of the ITO glasses is illustrated in Fig. 5, showing the scratch distance of the ITO glass without PEDOT:PSS film is $2.15 \mathrm{~mm}$ and increases to 2.26, 2.30, $2.32 \mathrm{~mm}$ as adding $30,40,50 \mathrm{~nm}$ thick PEDOT:PSS films. Similarly, the scratch distance of the ITO glasses is illustrated in Fig. 6 which shows the scratch distance of the ITO glass without PEDOT:PSS film is $166.0 \mathrm{mN}$ and then increases to $172.3,182.3,192.3 \mathrm{mN}$ as adding 30, 40, $50 \mathrm{~nm}$ thick PEDOT:PSS films, respectively. It reveals that the critical load of the ITO glass with PEDOT:PSS is higher than that without PEDOT:PSS. In average, the PEDOT:PSS increases $10 \%$ critical load of the ITO film. The scratch results indicate that the PEDOT:PSS film can improve the adhesion between the ITO thin film and the glass substrate.

Furthermore, all experimental results are listed in Table 2, including the surface roughness and electrical resistance. It indicates the surface roughness of the ITO glass without PEDOT:PSS film is $11.0 \mathrm{~nm}$, and decreases to $8.62,6.26$, and $5.36 \mathrm{~nm}$ as adding 30,40 , and $50 \mathrm{~nm}$ thick PEDOT:PSS films, respectively. It also indicates the sheet resistance of the ITO glass without PEDOT:PSS film is $13.77 \Omega / \square$, and decreases

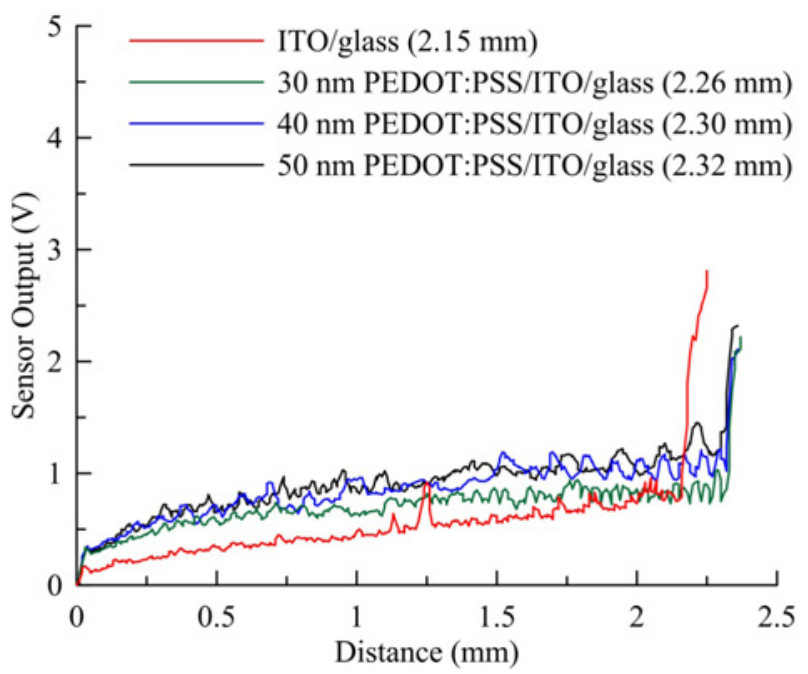

Fig. 5 Scratch distance of ITO glass with various thickness PEDOT:PSS films

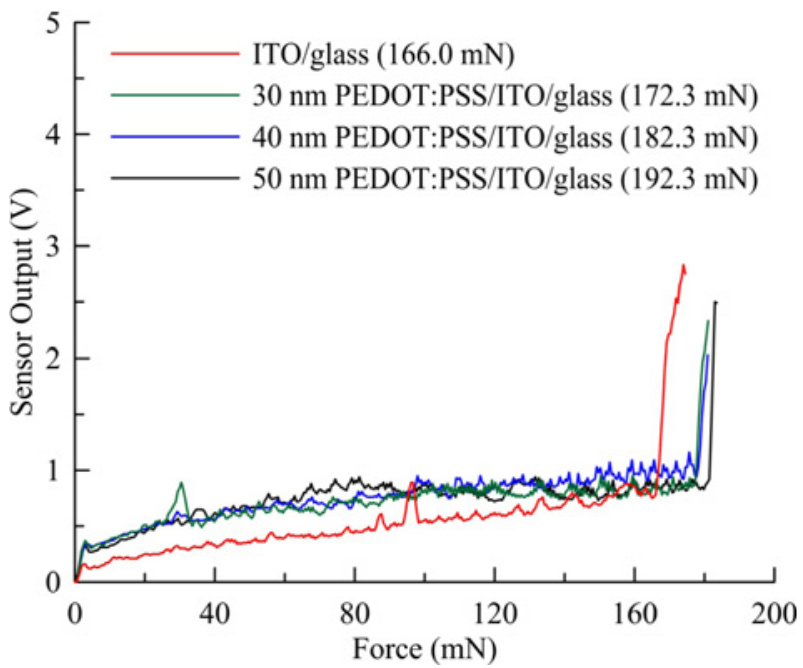

Fig. 6 Scratch force of ITO glass with various thickness PEDOT:PSS films 
to $5.28,4.78$, and $4.35 \Omega / \square$ as adding 30,40 , and $50 \mathrm{~nm}$ thick PEDOT:PSS films, respectively. In average, the surface roughness decreases $39 \%$ and the sheet resistance decreases $65 \%$ by adding the PEDOT:PSS film. It emphasizes the PEDOT:PSS film effectively improves the surface roughness and results in a lower electrical resistance of ITO glasses. In organic electronic devices, the sheet resistance of the ITO anode should be as low as possible. Kim et al. ${ }^{22}$ reported that the electric properties of ITO can be significantly modified by smooth surface. They also indicated that the presence of a PEDOT:PSS hole-transport layer inserted between the ITO anode and the emissive polymer layer is beneficial for the devices such as organic photovoltaic cells and light-emitting diodes. It is consistent with the results of this work.

\section{Conclusions}

The effects of various thickness PEDOT:PSS films on the performance of ITO glasses are discussed. Three thicknesses PEDOT:PSS are deposited on the ITO glass substrate by spin coating. The microstructure, mechanical properties, optical transmittance, and electric resistance of the PEDOT:PSS coated ITO glasses are tested in sequence. The results show that, comparing to the bare ITO glass, the PEDOT:PSS coated ITO glass improve 17\% residual stress, $10 \%$ adhesion, $39 \%$ roughness, and $65 \%$ electric resistance of the ITO glass. However, $1 \%$ transmittance, $49 \%$ modulus, and $89 \%$ hardness of the ITO glass are lost by adding the PEDOT:PSS films. In conclusion, the high conductivity and good adhesion indicate the compound of PEDOT:PSS and ITO will continue to play an important role in the applications of organic photovoltaic cells and light-emitting diodes.

\section{ACKNOWLEDGEMENT}

The authors would like to thank the support of the National Science Council through Grant NSC-102-2221-E-129-002-MY3.

\section{REFERENCES}

1. Granqvist, C. G. and Hultåker, A., "Transparent and Conducting ITO Films: New Developments and Applications," Thin Solid Films, Vol. 411, No. 1, pp. 1-5, 2002.

2. Tak, Y. H., Kim, K. B., Park, H. G., Lee, K. H., and Lee, J. R., "Transparent and Conducting ITO Films: New Developments and Applications,” Thin Solid Films, Vol. 411, No. 1, pp. 12-16, 2002.

3. Hoppe, H. and Sariciftci, N. S., "Organic Solar Cells: An Overview," Journal of Materials Research, Vol. 19, No. 7, pp. 19241945, 2004.

4. Shinar, J., "Organic Light-Emitting Devices: A Survey," SpringerVerlag, 2004.

5. Moujoud, A., Oh, S. H., Heo, K. Y., Lee, K. W., and Kim, H. J., "Indium Zinc Oxide Ohmic Contact to $\operatorname{Poly}(3,4-$ ethylenedioxythiophene) Poly(styrenesulfonate) Induced by UV Light,” Organic Electronics, Vol. 10, No. 5, pp. 785-790, 2009.

6. Nguyen, T., Le Rendu, P., Long, P., and De Vos, S., "Chemical and Thermal Treatment of PEDOT: PSS Thin Films for Use in Organic Light Emitting Diodes," Surface and Coatings Technology, Vol. 180-181, pp. 646-649, 2004.

7. Aernouts, T., Vanlaeke, P., Geens, W., Poortmans, J., Heremans, P., et al., "Printable Anodes for Flexible Organic Solar Cell Modules," Thin Solid Films, Vol. 451, pp. 22-25, 2004.

8. Kim, W., Kushto, G., Kim, H., and Kafafi, Z., "Effect of Annealing on the Electrical Properties and Morphology of a Conducting Polymer used as an Anode in Organic Light-Emitting Devices," Journal of Polymer Science Part B: Polymer Physics, Vol. 41, No. 21, pp. 2522-2528, 2003.

9. Mäkinen, A., Hill, I., Shashidhar, R., Nikolov, N., and Kafafi, Z., "Hole Injection Barriers at Polymer Anode/Small Molecule Interfaces," Applied Physics Letters, Vol. 79, No. 5, pp. 557-559, 2001.

10. Xiao B. W., Shang, Y. F., Meng, M., and Li, C. N., "Enhancement of Hole Injection with an Ultra-Thin $\mathrm{Ag}_{2} \mathrm{O}$ Modified Anode in Organic Light-Emitting Diodes," Microelectronics Journal, Vol. 36, No. 2, pp. 105-108, 2005.

11. Li, F., Chen, Z., Liu, C., and Gong, Q., "Improvement in Performance of Organic Light-Emitting Diodes by Adjusting Charge-Carrier Mobility in Organic/Inorganic Hybrid Hole Transporting Layer," Chemical Physics Letters, Vol. 412, No. 4, pp. 331-335, 2005.

12. Wang, G.-F., Tao, X.-M., and Wang, R.-X., "Fabrication and Characterization of OLEDs using PEDOT: PSS and MWCNT Nanocomposites," Composites Science and Technology, Vol. 68, No. 14, pp. 2837-2841, 2008.

13. Chiang, C.-J., Bull, S., Winscom, C., and Monkman, A., "A NanoIndentation Study of the Reduced Elastic Modulus of $\mathrm{Alq}_{3}$ and NPB Thin-Film Used in OLED Devices," Organic Electronics, Vol. 11, No. 3, pp. 450-455, 2010.

14. Guillen, C. and Herrero, J., "Comparison Study of ITO Thin Films Deposited by Sputtering at Room Temperature onto Polymer and Glass Substrates," Thin Solid Films, Vol. 480-481, pp. 129-132, 2005.

15. Kim, E.-H., Yang, C.-W., and Park, J.-W., "Improving the Delamination Resistance of Indium Tin Oxide (ITO) Coatings on Polymeric Substrates by $\mathrm{O}_{2}$ Plasma Surface Treatment," Current Applied Physics, Vol. 10, No. 3, pp. S510-S514, 2010.

16. Noyan, I. C. and Cohen, J. B., "Residual Stress: Measurement by Diffraction and Interpretation,” Springer-Verlag, 1987.

17. Welzel, U., Ligot, J., Lamparter, P., Vermeulen, A., and Mittemeijer, E., "Stress Analysis of Polycrystalline Thin Films and Surface Regions by X-Ray Diffraction,” Journal of Applied Crystallography, Vol. 38, No. 1, pp. 1-29, 2005. 
18. Oliver, W. C. and Pharr, G. M., "An Improved Technique for Determining Hardness and Elastic Modulus using Load and Displacement Sensing Indentation Experiments," Journal of Materials Research, Vol. 7, No. 6, pp. 1564-1583, 1992.

19. Sneddon, I. N., "The Relation between Load and Penetration in the Axisymmetric Boussinesq Problem for a Punch of Arbitrary Profile," International Journal of Engineering Science, Vol. 3, No. 1, pp. 47-57, 1965.

20. Beake, B. D., Ogwu, A. A., and Wagner, T., "Influence of Experimental Factors and Film Thickness on the Measured Critical Load in the Nanoscratch Test," Materials Science and Engineering: A, Vol. 423, No. 1, pp. 70-73, 2006.

21. Gorjanc, T., Leong, D., Py, C., and Roth, D., "Room Temperature Deposition of ITO using R.F. Magnetron Sputtering," Thin Solid Films, Vol. 413, No. 1, pp. 181-185, 2002.

22. Kim, J.-S., Cacialli, F., and Friend, R., "Surface Conditioning of Indium-Tin Oxide Anodes for Organic Light-Emitting Diodes," Thin Solid Films, Vol. 445, No. 2, pp. 358-366, 2003. 